\title{
OUTRANKING METHODS: PROMETHEE I AND PROMETHEE II
}

\section{Shankha Shubhra GOSWAMI}

Indira Gandhi Institute of Technology, Sarang, Dhenkanal, Odisha, INDIA e-mail: ssg.mech.official@gmail.com

\begin{abstract}
This article highlights the application of the Preference Ranking Organization Method for Enrichment of Evaluations (PROMETHEE) I and II in selecting the best laptop model among six different available models in the market. Seven important criteria, that is, processor, hard disk capacity, operating system, RAM, screen size, brand, and color, are selected, based on which the selection process have been made. Analytic hierarchy process (AHP) is adopted for calculating the weightages of the seven criteria and PROMETHEE is applied to select the best alternative. PROMETHEE I provides the partial ranking and preferences of one model over another, whereas PROMETHEE II provides the complete ranking of the alternatives. From this analysis, Model 4 is coming out to be the best laptop model occupying the first position and Model 1 occupies the last position, thus indicating it as the worst model among the group. The objectives of this article are to select the best laptop model among six available alternatives and to understood the steps of both multiple criteria decision-making (MCDM) methodologies, that is, PROMETHEE and AHP, in details.
\end{abstract}

Keywords: PROMETHEE I, PROMETHEE II, AHP, MCDM, laptop models.

JEL Classification: C61, C83, D81, M11.

\section{Introduction}

In this 21st century, computer has become one of the most essential electronics gadgets in our daily life. It makes our life easier and faster as compared to 15 years ago. In 2018, approximately 259.39 million of PCs were shipped around the world (Holst, 2019). Table 1 shows the global PC units' shipment from 2006 to 2018 (Statista, 2019).

Table 1. Global PC units' shipment from 2006-2018 (Source: Statista, 2019)

\begin{tabular}{|c|c|}
\hline Year & Shipments in millions \\
\hline 2018 & 259.39 \\
\hline 2017 & 262.68 \\
\hline 2016 & 269.72 \\
\hline 2015 & 287.68 \\
\hline 2014 & 313.68 \\
\hline 2013 & 316.46 \\
\hline 2012 & 351.06 \\
\hline 2011 & 365.36 \\
\hline 2010 & 350.90 \\
\hline 2009 & 308.34 \\
\hline 2008 & 290.80 \\
\hline 2007 & 272.45 \\
\hline 2006 & 239.21 \\
\hline
\end{tabular}

Moreover, with the technological development laptops are replacing the desktop computers and occupying the markets more rapidly because of their portability and ease of use. At present, there are six major laptop manufacturing companies in the market, that is, Lenovo, HP, Dell, Acer, Asus, and Apple. These brands have thousands of models out there in the market with different specifications, so it 
is very much important to select the appropriate laptop model so that most of our requirements can be fulfilled. Such an initiative is taken and presented in this article, where the best laptop model is proposed among six different models actually available in the market from different brands having different specifications by applying outranking methods the Preference Ranking Organization Method for Enrichment of Evaluations (PROMETHEE) I and II. The selected six laptop models and their specifications are presented in Table 2.

In this present analysis, six different laptop models having different configurations are selected from the market, which are presented in Table 2, and the aim of this research work is to propose the best model among them based on seven major criteria, that is, processor, hard disk capacity, operating system, RAM, screen size, brand, and color. After doing some research work, it is found that these models are in high demand and are generally preferred by most of the customers. The weightages of the criteria are found by analytic hierarchy process (AHP) and then PROMETHEE is applied by using those weightages to select the best model. Two categories of PROMETHEE are used in this article, where PROMETHEE I depicts the preferences of one model over another and PROMETHEE II helps to propose the complete ranking of the alternatives indicating from best to worst model.

Table 2. Selected laptop models and their configurations

(Source: Online shopping Websites and electronic stores)

\begin{tabular}{|c|c|c|c|c|c|c|c|}
\hline Models & Processor & $\begin{array}{c}\text { Hard Disk } \\
\text { Capacity }\end{array}$ & $\begin{array}{c}\text { Operating } \\
\text { System }\end{array}$ & RAM & Screen Size & Brand & Color \\
\hline Model 1 & I3 & $512 \mathrm{~GB}$ & DOS & 4GB & 14 Inch & HP & Black \\
\hline Model 2 & I5 & 1TB & Linux & $4 \mathrm{~GB}$ & 15.6 Inch & Acer & Black \\
\hline Model 3 & I5 & 2TB & Windows & $8 \mathrm{~GB}$ & 15.6 Inch & Lenovo & Gold \\
\hline Model 4 & I7 & 2TB & Windows & $16 \mathrm{~GB}$ & 17.3 Inch & Asus & Silver \\
\hline Model 5 & I5 & 1TB & Windows & $8 \mathrm{~GB}$ & 15.6 Inch & HP & Silver \\
\hline Model 6 & I3 & 512GB & Linux & $4 \mathrm{~GB}$ & 15.6 Inch & Dell & Black \\
\hline
\end{tabular}

\section{$2 \quad$ Literature Review}

For many years, multiple criteria decision-making (MCDM) proves to be a very useful tools for taking effective decision in the fields of water management (Qin, et al., 2008), waste management (Chandrakar and Limje, 2018), industries (Bentes, et al., 2012; Bulut, et al., 2012; Celik, et al., 2009; Duran and Aguilo, 2008; Roistamzadeh and Sofian, 2011; Sri Krishna, et al., 2014), business and finance (Albadvi, et al., 2007; Korhonen, et al., 2006; Lee, et al., 2008), medical and health sector (Buyukozkan and Cifci, 2012), education (Bhattacharya and Chakraborty, 2014; Melon, et al., 2008), environmental management (Geldermann, et al., 2000; Marzouk and Abdelakder, 2019; Vaillancourt and Waaub, 2004), energy management (Kowalski, et al., 2009; Tsoutsos, et al., 2009), and so on. Many researchers implemented various MCDM techniques in different areas for strategic decision making.
Some of the most popular MCDM techniques include AHP (Saaty, 1980; 2001; 2008; 2010), PROMETHEE (Brans, 1982; Brans, et al., 1984; 1986; Brans and Vincke, 1985), ELimination Et Choix Traduisant la REalité (ELECTRE) (Roy, 1968), Multi-Objective Optimization on the basis of Ratio Analysis (MOORA) (Brauers and Zavadskas, 2006; 2009), The Technique for Order of Preference by Similarity to Ideal Solution (TOPSIS) (Hwang, et al., 1993; Hwang and Yoon, 1981; Yoon, 1987), COmplex PRroportional ASsessment (COPRAS) (Zavadskas, et al., 2008), Additive Ratio ASsessment (ARAS) (Zavadskas, et al., 2010; Zavadskas and Turkis, 2010), and Fuzzy Analytic Hierarchy Process (FAHP) (Ayhan, 2013; Buckley, 1985; Chang, 1996; Zadeh, 1965). This section mainly includes some literatures (Behzadian, et al., 2010; Jayant and Sharma, 2018) of the researchers adopting PROMETHEE and AHP methods for decision-making purposes in various fields. 
Geldermann, et al. (2000) considered a case study in iron- and steel-making industry where the outranking method PROMETHEE was adopted for environmental assessment. Goumas and Lygerou (2000) applied PROMETHEE method for the evaluation and ranking of alternative energy exploitation schemes of a low-temperature geothermal field under fuzzy environment. Ngai and Chan (2005) applied analytic hierarchy process to select the most appropriate support knowledge management tool. Bertolini, et al. (2006) selected the best discount in defining a proposal for a public work contract by implementing AHP as a tool. Albadvi, et al. (2007) developed a decision-making model in their article for selecting superior stocks in stock exchange by using PROMETHEE method. Wang and Yang (2007) evaluated the information systems (IS) outsourcing problems using hybrid approach of AHP and PROMETHEE by considering six factors, where AHP was used to analyze the structure of the outsourcing problem and determine weights of the criteria and PROMETHEE method was used for final ranking of the alternatives.

Melon, et al. (2008) adopted AHP to evaluate the proposals for educational innovation projects to help the Institute of Educational Sciences of the Polytechnic University of Valencia to choose the best educational project. Tsoutsos, et al. (2009) determined a set of energy planning alternatives based on economic, technical, social, and environmental criteria factors by implementing PROMETHEE methodology for the sustainable energy planning on the island of Crete in Greece. Alomoush (2010) selected the best optimal location of thyristorcontrolled series compensator (TCSC) in a transmission system using PROMETHEE MCDM process. Turcksin, et al. (2011) proposed an integrated approach of AHP and PROMETHEE for selecting the most appropriate policy scenario to stimulate a clean vehicle fleet. Yilmaz and Dagdeviren (2011) used a combined approach of fuzzy-PROMETHEE and zero-one goal programming model for solving welding machine selection problem of a company.

Peng and Xiao (2013) considered a material selection problem for a journal bearing in which PROMETHEE combined with analytic network process (ANP) is presented; ANP was used to identify the weights and PROMETHEE was used to rank alternatives. Zhao, et al. (2013) proposed a modified PROMETHEE method to improve the efficiency and response time in incident management. Kabir and Sumi (2014) considered a case study from Bangladesh where fuzzy-AHP and PROMETHEE were used to select the power substation location. $\mathrm{Ku}$ charski (2014) used PROMETHEE method to rank selected types of investment funds offered on the polish market. Maletic, et al. (2014) applied AHP for the selection of maintenance policy. Kilic, et al. (2015) performed an analysis on the enterprise resource planning (ERP) selection problem for the small medium enterprises (SMEs) in Istanbul, Turkey, by combining AHP-PROMETHEE hybrid MCDM methodology.

Polat (2016) used integrated AHP-PROMETHEE methods for selecting the most appropriate subcontractor to be worked within an international construction project, where AHP was used to analyze the structure of the subcontractor selection problem and to determine the weights of the criteria and PROMETHEE was used to obtain complete ranking and perform sensitivity analysis by changing the weights of criteria. Polat, et al. (2016) proposed an integrated approach of AHP and PROMETHEE to help a Turkish construction company in selecting the appropriate urban renewal project by finding the weights of the selected criteria and to rank the alternative projects, respectively. Nikouei, et al. (2017) used PROMETHEE based on MCDM approach for selecting the best membrane prepared from sulfonated poly(ether ketone)s and poly(ether sulfone)s, and the final results showed that poly(ether ketone) membranes in selected criteria were better than poly(ether sulfone) membranes. Nourbakhsh and Yousefi (2017) proposed the best indicator water quality parameters using AHP in order to manage the ground water quality. Butowski (2018) developed an integrated AHP and PROMETHEE approach to evaluate the attractiveness of European maritime areas for sailing tourism. Naserizade, et al. (2018) developed a new stochastic model based on conditional value at risk ( $\mathrm{CVaR})$ and multi-objective optimization methods for optimal placement of sensors in water distribution system (WDS), and finally, PROMETHEE was used to de- 
termine the best solution and to rank the alternatives on the trade-off curve among objective functions.

Apart from the above literatures, there are also few research works in which MCDM techniques are being used for selecting electronics devices, household appliances or other items that are associated with our daily life, for example, selection of desktop (Mitra and Goswami, 2019a; 2019b) and laptop computers (Adali and Isik, 2017), selection of mobiles (Goswami and Mitra, 2020), car selection (Sri Krishna, et al., 2014), refrigerator selection (Mitra and Kundu, 2017; 2018), and air conditioner selection (Adali and Isik, 2016), that have been recorded but there are very few researchers who have adopted and applied PROMETHEE methods for solving decision-making problems associated with our daily life apart from industrial applications. So, such an initiative is taken in this article to apply an integrated AHPPROMETHEE (Butowski, 2018; Kilic, et al., 2015; Polat, et al., 2016; Turcksin, et al., 2011; Wang and Yang, 2007) methodologies to solve the laptop selection problems, where AHP is used for calculating the criteria weightages and PROMETHEE is used for the final ranking of the alternatives.

\section{$3 \quad$ Materials and Methods}

This section consists of all the step-wise calculation details of AHP and PROMETHEE. The weightages of the criteria are calculated using AHP, and the final ranking of the alternatives are performed using PROMETHEE, which are discussed in Sections 3.1 and 3.2 , respectively.

\subsection{Analytic Hierarchy Process (AHP)}

AHP is an MCDM technique for establishing and analyzing complex decisions based on psychology and mathematics (Saaty, 1980; 2001; 2008; 2010). It was first developed by professor Thomas L. Saaty in 1980 (Saaty, 1980). The AHP methodology is illustrated briefly in Fig. 1.

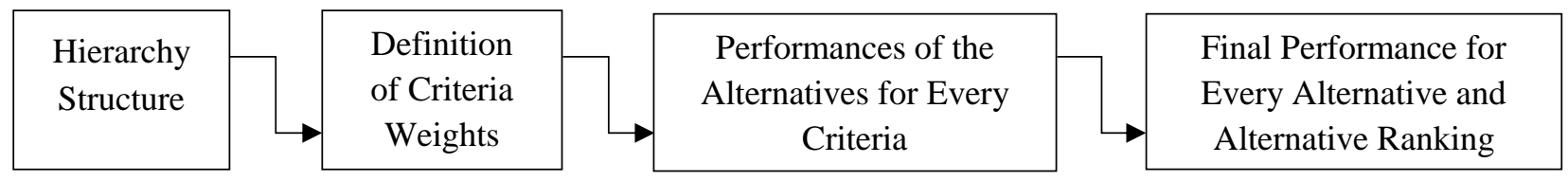

Figure 1. AHP methodology (Source: Kolios, et al., 2016)

The steps involved in AHP for calculating the criteria weightages are given as follows:
Step 1: An $n \times n$ pair-wise comparison matrix is created based on Saaty's 9 pair comparison scale (Saaty, 1980), which is presented in Table 3.

Table 3. Pair-wise comparison matrix (Source: Own elaboration)

\begin{tabular}{|c|c|c|c|c|c|c|c|}
\hline Comparisons & Processor & $\begin{array}{c}\text { Hard disk } \\
\text { capacity }\end{array}$ & $\begin{array}{c}\text { Operating } \\
\text { system }\end{array}$ & RAM & $\begin{array}{c}\text { Screen } \\
\text { size }\end{array}$ & Brand & Color \\
\hline Processor & 1 & 5 & 7 & 3 & 2 & 7 & 9 \\
\hline Hard disk capacity & $1 / 5$ & 1 & 3 & $1 / 3$ & $1 / 3$ & 2 & 5 \\
\hline Operating system & $1 / 7$ & $1 / 3$ & 1 & $1 / 5$ & $1 / 3$ & $1 / 3$ & 3 \\
\hline RAM & $1 / 3$ & 3 & 5 & 1 & 2 & 3 & 9 \\
\hline Screen size & $1 / 2$ & 3 & 3 & $1 / 2$ & 1 & 3 & 7 \\
\hline Brand & $1 / 7$ & $1 / 2$ & 3 & $1 / 3$ & $1 / 3$ & 1 & 5 \\
\hline Color & $1 / 9$ & $1 / 5$ & $1 / 3$ & $1 / 9$ & $1 / 7$ & $1 / 5$ & 1 \\
\hline Total & $\mathbf{2 . 4 3 0 1 6}$ & $\mathbf{1 3 . 0 3 3 3 3}$ & $\mathbf{2 2 . 3 3 3 3 3}$ & $\mathbf{5 . 4 7 7 7 8}$ & $\mathbf{6 . 1 4 2 8 6}$ & $\mathbf{1 6 . 5 3 3 3 3}$ & $\mathbf{3 9}$ \\
\hline
\end{tabular}


This pair-wise comparison matrix compares all the criteria among each other, and the relative importance of one criterion over other is given according to the Saaty pair-wise comparison scale.

Table 5 presents the Saaty's 9 pair comparison scale. Here, $\mathrm{n}$ is the number of criteria considered for the analysis. In this case, seven criteria are considered; hence, $\mathrm{n}=7$.

Step 2: The pair-wise comparison matrix (presented in Table 3) is normalized by dividing all the elements of Table 3 by their respective column sum. Table 4 presents the normalized pair-wise comparison matrix.

Table 4. Normalized pair-wise comparison matrix (Source: Own elaboration)

\begin{tabular}{|c|c|c|c|c|c|c|c|c|c|}
\hline Comparisons & Processor & $\begin{array}{c}\text { Hard disk } \\
\text { capacity }\end{array}$ & $\begin{array}{c}\text { Operating } \\
\text { system }\end{array}$ & RAM & $\begin{array}{c}\text { Screen } \\
\text { size }\end{array}$ & Brand & Color & $\begin{array}{c}\text { Priority } \\
\text { Vector }\end{array}$ & $\begin{array}{c}\text { Weight } \\
\%\end{array}$ \\
\hline Processor & 0.41150 & 0.38363 & 0.31343 & 0.54767 & 0.32558 & 0.42339 & 0.23077 & 0.37657 & 37.657 \\
\hline $\begin{array}{c}\text { Hard disk } \\
\text { capacity }\end{array}$ & 0.08230 & 0.07673 & 0.13433 & 0.06085 & 0.05426 & 0.12097 & 0.12821 & 0.09395 & 9.395 \\
\hline $\begin{array}{c}\text { Operating } \\
\text { system }\end{array}$ & 0.05879 & 0.02558 & 0.04478 & 0.03651 & 0.05426 & 0.02016 & 0.07692 & 0.04529 & 4.529 \\
\hline RAM & 0.13717 & 0.23018 & 0.22388 & 0.18256 & 0.32558 & 0.18145 & 0.23077 & 0.21594 & 21.594 \\
\hline Screen size & 0.20575 & 0.23018 & 0.13433 & 0.09128 & 0.16279 & 0.18145 & 0.17949 & 0.16932 & 16.932 \\
\hline Brand & 0.05879 & 0.03836 & 0.13433 & 0.06085 & 0.05426 & 0.06048 & 0.12821 & 0.07647 & 7.647 \\
\hline Color & 0.04572 & 0.01535 & 0.01493 & 0.02028 & 0.02326 & 0.01210 & 0.02564 & 0.02247 & 2.247 \\
\hline Total & $\mathbf{1}$ & $\mathbf{1}$ & $\mathbf{1}$ & $\mathbf{1}$ & $\mathbf{1}$ & $\mathbf{1}$ & $\mathbf{1}$ & $\mathbf{1}$ & $\mathbf{1 0 0}$ \\
\hline
\end{tabular}

The normalized values are calculated and presented in Table 4. All the row averages (priority vector) are found out for every criterion, which are the criteria weightages. The criteria weightages are found out to be as follows: $\mathrm{w}_{\text {processor }}=0.37657$ or $37.657 \%$, $\mathrm{w}_{\text {hard disk capacity }}=0.09395$ or $9.395 \%, \mathrm{w}_{\text {operating system }}=$ 0.04529 or $4.529 \%, \mathrm{w}_{\mathrm{RAM}}=0.21594$ or $21.594 \%$, $\mathrm{W}_{\text {screen size }}=0.16932$ or $16.932 \%, \mathrm{w}_{\text {brand }}=0.07647$ or $7.647 \%$, and $\mathrm{w}_{\text {color }}=0.02247$ or $2.247 \%$.
Table 4 presents the normalized decision matrix along with the criteria weightages. Now the next step is to check the consistency whether the decision maker's judgment is true and consistent.

Step 3: Calculate the average consistency $\left(\lambda_{\max }\right)$. The priority vector matrix (priority vector column in Table 4) is multiplied with the pair-wise comparison matrix (Table 3 ) and $\lambda_{\max }$ is calculated as shown in details below:

$$
\begin{gathered}
{\left[\begin{array}{ccccccc}
1 & 5 & 7 & 3 & 2 & 7 & 9 \\
1 / 5 & 1 & 3 & 1 / 3 & 1 / 3 & 2 & 5 \\
1 / 7 & 1 / 3 & 1 & 1 / 5 & 1 / 3 & 1 / 3 & 3 \\
1 / 3 & 3 & 5 & 1 & 2 & 3 & 9 \\
1 / 2 & 3 & 3 & 1 / 2 & 1 & 3 & 7 \\
1 / 7 & 1 / 2 & 3 & 1 / 3 & 1 / 3 & 1 & 5 \\
1 / 9 & 1 / 5 & 1 / 3 & 1 / 9 & 1 / 7 & 1 / 5 & 1
\end{array}\right] \times\left[\begin{array}{c}
0.37657 \\
0.09395 \\
0.04529 \\
0.21594 \\
0.16932 \\
0.07647 \\
0.02247
\end{array}\right]=\left[\begin{array}{l}
2.88726 \\
0.69881 \\
0.32292 \\
1.61999 \\
1.26995 \\
0.55385 \\
0.16167
\end{array}\right]} \\
\left|\begin{array}{l}
2.88726 / 0.37657 \\
0.69881 / 0.09395 \\
0.32292 / 0.04529 \\
1.61999 / 0.21594 \\
1.26995 / 0.16932 \\
0.55385 / 0.07647 \\
0.16167 / 0.02247
\end{array}\right|=\left|\begin{array}{c}
7.66733 \\
7.43821 \\
7.13075 \\
7.50203 \\
7.50018 \\
7.24284 \\
7.19579
\end{array}\right| \quad \begin{array}{c}
\text { Processor } \\
\text { Hard disk capacity } \\
\text { Operating system } \\
\text { RAM } \\
\text { Screen size } \\
\text { Brand } \\
\text { Color }
\end{array}
\end{gathered}
$$


The consistencies of all the seven criteria are found out. Now, calculate the averages of these seven values to find out the average consistency $\left(\lambda_{\max }\right)$.

Average consistency

$\left(\lambda_{\max }\right)=$

$7.66733+7.43821+7.13075+7.50203+7.50018+7.24284+7.19579$ $=7.38245$.

Step 4: Calculate the consistency ratio (CR) value and check for consistency. $\mathrm{CR}$ value is calculated using Equation 1 as shown below.

$$
\text { Consistency ratio }(\mathrm{CR})=\frac{\mathrm{CI}}{\mathrm{RI}}
$$

where $\mathrm{CI}$ is the consistency index and RI is the randomly generated $\mathrm{CI}$.
CI is calculated using Equation 2, and RI value can be obtained from Table 6 according to the number criteria. In this case, $\mathrm{n}=7$, so the RI value corresponding to $\mathrm{n}=7$ is 1.32 .

$$
\text { Consistency Index }(\mathrm{CI})=\frac{\left(\lambda_{\max }-\mathrm{n}\right)}{(\mathrm{n}-1)}
$$

Here, $\lambda_{\max }$ is the average consistency and $n$ is the number of criteria. In this case, $n=7$.

Using Equations 1 and 2, CI and CR values are found to be 0.06374 (CI value) and 0.04829 (CR value) or $4.829 \%$.

Here it can be seen that the CR value is less than 0.1 $(0.04829<0.1)$, that is, the inconsistency is within $10 \%$; hence, it can be concluded that the decision maker judgment is true and consistent.

Table 5. Saaty's pair-wise comparison scale (Source: Saaty, 1980)

\begin{tabular}{|c|c|}
\hline Saaty's pair wise comparison scale & Compare factor of $\mathrm{i} \& \mathrm{j}$ \\
\hline 1 & Equal Importance \\
\hline 3 & Moderate Importance \\
\hline 5 & Strong Importance \\
\hline 7 & Very Strong or Demonstrated Importance \\
\hline 9 & Extreme Importance \\
\hline $2,4,6,8$ & Intermediate values when compromise is needed \\
\hline
\end{tabular}

Table 6. Randomly generated consistency index (RI) values (Source: Saaty, 1980)

\begin{tabular}{|c|c|c|c|c|c|c|c|c|c|c|c|c|}
\hline $\mathrm{n}$ & 1 & 2 & 3 & 4 & 5 & 6 & 7 & 8 & 9 & 10 & 11 & 12 \\
\hline $\mathrm{RI}$ & 0.00 & 0.00 & 0.58 & 0.90 & 1.12 & 1.24 & 1.32 & 1.41 & 1.45 & 1.49 & 1.51 & 1.58 \\
\hline
\end{tabular}

\subsection{Preference Ranking Organization Method for Enrichment of Evaluations}

In early 1980s, for the first time, Brans presented the PROMETHEE outranking method in 1982 at a conference organized by R. Nadeau and M. Landry at the University Laval, Québec, Canada (Brans,
1982). After that, developments have been carried out for more than a decade to bring out various versions of PROMETHEE by Brans along with other researchers (Brans, 1982; Brans, et al., 1984, 1986; Brans and Mareschal, 1992, 1994, 1995; Brans and Vincke, 1985). The PROMETHEE methodology are explained briefly in Fig. 2.

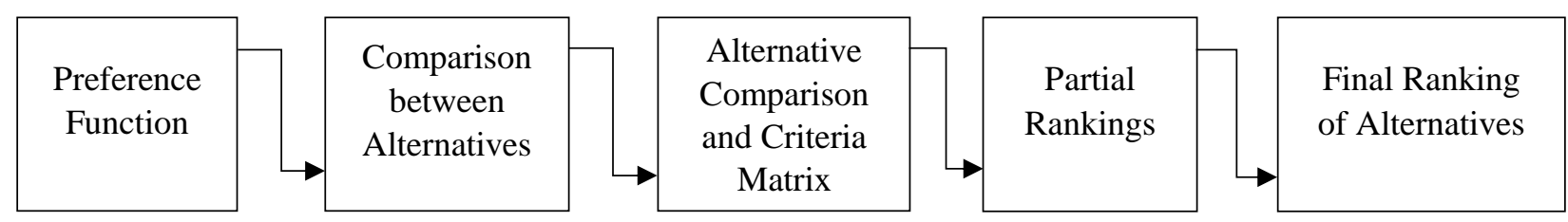

Figure 2. PROMETHEE methodology (Source: Kolios, et al., 2016) 
The steps involved in PROMETHEE are described as follows.

Step 1: Create an $\mathrm{m} \times \mathrm{n}$ evaluation (decision) matrix according to Equation 3 based on the Hwang and Yoon comparison scale (Hwang and Yoon, 1981),

$$
X=\left[\mathrm{x}_{\mathrm{ij}}\right]_{\mathrm{m} \times \mathrm{n}}=\left[\begin{array}{cccc}
\mathrm{x}_{11} & \mathrm{x}_{12} & \cdots & \mathrm{x}_{1 \mathrm{n}} \\
\mathrm{x}_{21} & \mathrm{x}_{22} & \cdots & \mathrm{x}_{2 \mathrm{n}} \\
\cdots & \cdots & \cdots & \cdots \\
\mathrm{x}_{\mathrm{m} 1} & \mathrm{x}_{\mathrm{m} 2} & \cdots & \mathrm{x}_{\mathrm{mn}}
\end{array}\right]
$$

where $i=1,2,3 \ldots, m$ and $j=1,2,3 \ldots, n$. which is presented in Table 8, where $\mathrm{m}$ is the number of alternatives and $n$ is the number of criteria. Table 7 presents the decision matrix.

Table 7. Evaluation matrix (Source: Own elaboration)

\begin{tabular}{|c|c|c|c|c|c|c|c|}
\hline Models & Processor & $\begin{array}{c}\text { Hard disk } \\
\text { capacity }\end{array}$ & $\begin{array}{c}\text { Operating } \\
\text { system }\end{array}$ & RAM & $\begin{array}{c}\text { Screen } \\
\text { size }\end{array}$ & Brand & Color \\
\hline Model 1 & 3 & 5 & 3 & 5 & 3 & 9 & 3 \\
\hline Model 2 & 5 & 7 & 5 & 5 & 7 & 3 & 3 \\
\hline Model 3 & 5 & 9 & 9 & 7 & 7 & 7 & 5 \\
\hline Model 4 & 7 & 9 & 9 & 9 & 9 & 2 & 9 \\
\hline Model 5 & 5 & 7 & 9 & 7 & 7 & 9 & 9 \\
\hline Model 6 & 3 & 5 & 5 & 5 & 7 & 5 & 3 \\
\hline Max & 7 & 9 & 9 & 9 & 9 & 9 & 9 \\
\hline Min & 3 & 5 & 3 & 5 & 3 & 2 & 3 \\
\hline
\end{tabular}

Table 8. Hwang ang Yoon comparison scale (Source: Hwang and Yoon, 1981)

\begin{tabular}{|c|c|c|c|c|c|c|}
\hline Qualitative Estimation & Bad & Good & Average & Very Good & Excellent & Types of Criteria \\
\hline \multirow{2}{*}{ Quantitative Estimation } & 1 & 3 & 5 & 7 & 9 & Max \\
\cline { 2 - 7 } & 9 & 7 & 5 & 3 & 1 & Min \\
\hline
\end{tabular}

Step 2: The decision matrix presented in Table 7 is normalized using Equations 4 and 5 according to the nature of the selected criteria.

For beneficial criteria

$$
\mathrm{R}_{\mathrm{ij}}=\frac{\left[\mathrm{x}_{\mathrm{ij}}-\min \left(\mathrm{x}_{\mathrm{ij}}\right)\right]}{\left[\max \left(\mathrm{x}_{\mathrm{ij}}\right)-\min \left(\mathrm{x}_{\mathrm{ij}}\right)\right]},
$$

For Non-beneficial criteria or cost criteria

$$
\mathrm{R}_{\mathrm{ij}}=\frac{\left[\max \left(\mathrm{x}_{\mathrm{ij}}\right)-\mathrm{x}_{\mathrm{ij}}\right]}{\left[\max \left(\mathrm{x}_{\mathrm{ij}}\right)-\min \left(\mathrm{x}_{\mathrm{ij}}\right)\right]},
$$

where $i=1,2,3 \ldots m ; j=1,2,3 \ldots n$.

All the criteria considered for this analysis are beneficial in nature, that is, whose higher values are desired. So Equation 4 is used for the normalization of the decision matrix. Table 9 presents the normalized decision matrix.

Table 9. Normalized decision matrix (Source: Own elaboration)

\begin{tabular}{|c|c|c|c|c|c|c|c|}
\hline Models & Processor & $\begin{array}{c}\text { Hard disk } \\
\text { capacity }\end{array}$ & $\begin{array}{c}\text { Operating } \\
\text { system }\end{array}$ & RAM & $\begin{array}{c}\text { Screen } \\
\text { size }\end{array}$ & Brand & Color \\
\hline Model 1 & 0 & 0 & 0 & 0 & 0 & 1 & 0 \\
\hline Model 2 & 0.5 & 0.5 & 0.33333 & 0 & 0.66667 & 0.14286 & 0 \\
\hline Model 3 & 0.5 & 1 & 1 & 0.5 & 0.66667 & 0.71429 & 0.33333 \\
\hline Model 4 & 1 & 1 & 1 & 1 & 1 & 0 & 1 \\
\hline Model 5 & 0.5 & 0.5 & 1 & 0.5 & 0.66667 & 1 & 1 \\
\hline Model 6 & 0 & 0 & 0.33333 & 0 & 0.66667 & 0.42857 & 0 \\
\hline
\end{tabular}


Step 3: Calculate the evaluative differences of the $i^{\text {th }}$ alternative with respect to the other alternatives using Equation 6.
The evaluative differences (deviations) are calculated and presented in Table 10.

$\mathrm{D}(\mathrm{Ma}-\mathrm{Mb})=\left(\mathrm{R}_{(\mathrm{ij})_{\mathrm{a}}}-\mathrm{R}_{(\mathrm{ij})_{\mathrm{b}}}\right)$

Table 10. Evaluative difference of $\mathrm{i}^{\text {th }}$ alternatives with respect other alternatives (Source: Own elaboration)

\begin{tabular}{|c|c|c|c|c|c|c|c|}
\hline $\begin{array}{l}\text { Evaluative } \\
\text { difference }\end{array}$ & Processor & $\begin{array}{c}\text { Hard disk } \\
\text { capacity }\end{array}$ & $\begin{array}{c}\text { Operating } \\
\text { system }\end{array}$ & RAM & $\begin{array}{c}\text { Screen } \\
\text { size }\end{array}$ & Brand & Color \\
\hline \multicolumn{8}{|c|}{ Model 1} \\
\hline D (M1-M2) & -0.5 & -0.5 & -0.33333 & 0 & -0.66667 & 0.85714 & 0 \\
\hline D (M1-M3) & -0.5 & -1 & -1 & -0.5 & -0.66667 & 0.28571 & -0.33333 \\
\hline D (M1-M4) & -1 & -1 & -1 & -1 & -1 & 1 & -1 \\
\hline D (M1-M5) & -0.5 & -0.5 & -1 & -0.5 & -0.66667 & 0 & -1 \\
\hline D (M1-M6) & 0 & 0 & -0.33333 & 0 & -0.66667 & 0.57143 & 0 \\
\hline \multicolumn{8}{|c|}{ Model 2} \\
\hline D (M2-M1) & 0.5 & 0.5 & 0.33333 & 0 & 0.66667 & -0.85714 & 0 \\
\hline D (M2-M3) & 0 & -0.5 & -0.66667 & -0.5 & 0 & -0.57143 & -0.33333 \\
\hline D (M2-M4) & -0.5 & -0.5 & -0.66667 & -1 & -0.33333 & 0.14286 & -1 \\
\hline D (M2-M5) & 0 & 0 & -0.66667 & -0.5 & 0 & -0.85714 & -1 \\
\hline D (M2-M6) & 0.5 & 0.5 & 0 & 0 & 0 & -0.28571 & 0 \\
\hline \multicolumn{8}{|c|}{ Model 3} \\
\hline D (M3-M1) & 0.5 & 1 & 1 & 0.5 & 0.66667 & -0.28571 & 0.33333 \\
\hline D (M3-M2) & 0 & 0.5 & 0.66667 & 0.5 & 0 & 0.57143 & 0.33333 \\
\hline D (M3-M4) & -0.5 & 0 & 0 & -0.5 & -0.33333 & 0.71429 & -0.66667 \\
\hline D (M3-M5) & 0 & 0.5 & 0 & 0 & 0 & -0.28571 & -0.66667 \\
\hline D (M3-M6) & 0.5 & 1 & 0.66667 & 0.5 & 0 & 0.28571 & 0.33333 \\
\hline \multicolumn{8}{|c|}{ Model 4} \\
\hline D (M4-M1) & 1 & 1 & 1 & 1 & 1 & -1 & 1 \\
\hline D (M4-M2) & 0.5 & 0.5 & 0.66667 & 1 & 0.33333 & -0.14286 & 1 \\
\hline D (M4-M3) & 0.5 & 0 & 0 & 0.5 & 0.33333 & -0.71429 & 0.66667 \\
\hline D (M4-M5) & 0.5 & 0.5 & 0 & 0.5 & 0.33333 & -1 & 0 \\
\hline D (M4-M6) & 1 & 1 & 0.66667 & 1 & 0.33333 & -0.42857 & 1 \\
\hline \multicolumn{8}{|c|}{ Model 5} \\
\hline D (M5-M1) & 0.5 & 0.5 & 1 & 0.5 & 0.66667 & 0 & 1 \\
\hline D (M5-M2) & 0 & 0 & 0.66667 & 0.5 & 0 & 0.85714 & 1 \\
\hline D (M5-M3) & 0 & -0.5 & 0 & 0 & 0 & 0.28571 & 0.66667 \\
\hline D (M5-M4) & -0.5 & -0.5 & 0 & -0.5 & -0.33333 & 1 & 0 \\
\hline D (M5-M6) & 0.5 & 0.5 & 0.66667 & 0.5 & 0 & 0.57143 & 1 \\
\hline \multicolumn{8}{|c|}{ Model 6} \\
\hline D (M6-M1) & 0 & 0 & 0.33333 & 0 & 0.66667 & -0.57143 & 0 \\
\hline D (M6-M2) & -0.5 & -0.5 & 0 & 0 & 0 & 0.28571 & 0 \\
\hline D (M6-M3) & -0.5 & -1 & -0.66667 & -0.5 & 0 & -0.28571 & -0.33333 \\
\hline D (M6-M4) & -1 & -1 & -0.66667 & -1 & -0.33333 & 0.42857 & -1 \\
\hline D (M6-M5) & -0.5 & -0.5 & -0.66667 & -0.5 & 0 & -0.57143 & -1 \\
\hline
\end{tabular}


Step 4: Calculate the preference function $\mathrm{P}_{\mathrm{j}}$ (Ma, $\mathrm{Mb})$.

The preference function is calculated using the following two conditions given by Equations 7 and 8 .

$$
\begin{aligned}
& P_{j}(M a, M b)=0 \\
& \text { if } R_{(i j)_{a}} \leq R_{(i j)_{b}} \rightarrow D(M a-M b) \leq 0 \\
& P_{j}(M a, M b)=\left(R_{(i j)_{a}}-R_{(i j)_{b}}\right) \\
& \quad \text { if } R_{(i j)_{a}}>R_{(i j)_{b}} \rightarrow D(M a-M b)>0
\end{aligned}
$$

\begin{tabular}{|c|c|c|c|c|c|c|c|}
\hline Weights $\left(\mathrm{w}_{\mathrm{j}}\right)$ & 0.37657 & 0.09395 & 0.04529 & 0.21594 & 0.16932 & 0.07647 & 0.02247 \\
\hline $\begin{array}{l}\text { Evaluative dif- } \\
\text { ference }\end{array}$ & Processor & $\begin{array}{c}\text { Hard disk } \\
\text { capacity }\end{array}$ & $\begin{array}{c}\text { Operating } \\
\text { system }\end{array}$ & RAM & $\begin{array}{c}\text { Screen } \\
\text { size }\end{array}$ & Brand & Color \\
\hline \multicolumn{8}{|c|}{ Model 1} \\
\hline $\mathrm{P}(\mathrm{M} 1, \mathrm{M} 2)$ & 0 & 0 & 0 & 0 & 0 & 0.85714 & 0 \\
\hline $\mathrm{P}(\mathrm{M} 1, \mathrm{M} 3)$ & 0 & 0 & 0 & 0 & 0 & 0.28571 & 0 \\
\hline $\mathrm{P}(\mathrm{M} 1, \mathrm{M} 4)$ & 0 & 0 & 0 & 0 & 0 & 1 & 0 \\
\hline $\mathrm{P}(\mathrm{M} 1, \mathrm{M} 5)$ & 0 & 0 & 0 & 0 & 0 & 0 & 0 \\
\hline $\mathrm{P}(\mathrm{M} 1, \mathrm{M} 6)$ & 0 & 0 & 0 & 0 & 0 & 0.57143 & 0 \\
\hline \multicolumn{8}{|c|}{ Model 2} \\
\hline $\mathrm{P}(\mathrm{M} 2, \mathrm{M} 1)$ & 0.5 & 0.5 & 0.33333 & 0 & 0.66667 & 0 & 0 \\
\hline $\mathrm{P}(\mathrm{M} 2, \mathrm{M} 3)$ & 0 & 0 & 0 & 0 & 0 & 0 & 0 \\
\hline $\mathrm{P}(\mathrm{M} 2, \mathrm{M} 4)$ & 0 & 0 & 0 & 0 & 0 & 0.14286 & 0 \\
\hline $\mathrm{P}$ (M2, M5) & 0 & 0 & 0 & 0 & 0 & 0 & 0 \\
\hline $\mathrm{P}$ (M2, M6) & 0.5 & 0.5 & 0 & 0 & 0 & 0 & 0 \\
\hline \multicolumn{8}{|c|}{ Model 3} \\
\hline $\mathrm{P}(\mathrm{M} 3, \mathrm{M} 1)$ & 0.5 & 1 & 1 & 0.5 & 0.66667 & 0 & 0.33333 \\
\hline $\mathrm{P}(\mathrm{M} 3, \mathrm{M} 2)$ & 0 & 0.5 & 0.66667 & 0.5 & 0 & 0.57143 & 0.33333 \\
\hline $\mathrm{P}(\mathrm{M} 3, \mathrm{M} 4)$ & 0 & 0 & 0 & 0 & 0 & 0.71429 & 0 \\
\hline $\mathrm{P}(\mathrm{M} 3, \mathrm{M} 5)$ & 0 & 0.5 & 0 & 0 & 0 & 0 & 0 \\
\hline $\mathrm{P}$ (M3, M6) & 0.5 & 1 & 0.66667 & 0.5 & 0 & 0.28571 & 0.33333 \\
\hline \multicolumn{8}{|c|}{ Model 4} \\
\hline $\mathrm{P}(\mathrm{M} 4, \mathrm{M} 1)$ & 1 & 1 & 1 & 1 & 1 & 0 & 1 \\
\hline $\mathrm{P}(\mathrm{M} 4, \mathrm{M} 2)$ & 0.5 & 0.5 & 0.66667 & 1 & 0.33333 & 0 & 1 \\
\hline $\mathrm{P}(\mathrm{M} 4, \mathrm{M} 3)$ & 0.5 & 0 & 0 & 0.5 & 0.33333 & 0 & 0.66667 \\
\hline $\mathrm{P}$ (M4, M5) & 0.5 & 0.5 & 0 & 0.5 & 0.33333 & 0 & 0 \\
\hline $\mathrm{P}(\mathrm{M} 4, \mathrm{M} 6)$ & 1 & 1 & 0.66667 & 1 & 0.33333 & 0 & 1 \\
\hline \multicolumn{8}{|c|}{ Model 5} \\
\hline $\mathrm{P}(\mathrm{M} 5, \mathrm{M} 1)$ & 0.5 & 0.5 & 1 & 0.5 & 0.66667 & 0 & 1 \\
\hline $\mathrm{P}(\mathrm{M} 5, \mathrm{M} 2)$ & 0 & 0 & 0.66667 & 0.5 & 0 & 0.85714 & 1 \\
\hline $\mathrm{P}(\mathrm{M} 5, \mathrm{M} 3)$ & 0 & 0 & 0 & 0 & 0 & 0.28571 & 0.66667 \\
\hline $\mathrm{P}(\mathrm{M} 5, \mathrm{M} 4)$ & 0 & 0 & 0 & 0 & 0 & 1 & 0 \\
\hline $\mathrm{P}$ (M5, M6) & 0.5 & 0.5 & 0.66667 & 0.5 & 0 & 0.57143 & 1 \\
\hline \multicolumn{8}{|c|}{ Model 6} \\
\hline $\mathrm{P}(\mathrm{M} 6, \mathrm{M} 1)$ & 0 & 0 & 0.33333 & 0 & 0.66667 & 0 & 0 \\
\hline $\mathrm{P}(\mathrm{M} 6, \mathrm{M} 2)$ & 0 & 0 & 0 & 0 & 0 & 0.28571 & 0 \\
\hline P (M6, M3) & 0 & 0 & 0 & 0 & 0 & 0 & 0 \\
\hline P (M6, M4) & 0 & 0 & 0 & 0 & 0 & 0.42857 & 0 \\
\hline P (M6, M5) & 0 & 0 & 0 & 0 & 0 & 0 & 0 \\
\hline
\end{tabular}

Table 11. Preference function, $\mathrm{P}_{\mathrm{j}}(\mathrm{Ma}, \mathrm{Mb})$

(Source: Own elaboration) 
From Equations 7 and 8, it is clear that if the difference, that is, $\mathrm{D}(\mathrm{Ma}-\mathrm{Mb})$ in Table 10 is less than or equal to zero, then substitute the preference function value as zero and if $\mathrm{D}(\mathrm{Ma}-\mathrm{Mb})$ value is greater than zero, then the differences, that is, $\left(R_{(i j)_{a}}-\right.$ $R_{(i j)_{b}}$ ) is used as the preference function value.

In more easy words, if the value in any of the cell in Table 10 is less than or equal to zero (i.e., negative values), then substitute the value as zero and if the value in any of the cell in Table 10 is greater than zero, then leave it untouched. So, by substituting all the negative values of Table 10 by zeroes and keeping all the positive values as it is, thus obtaining the Table 11 as shown above.
Step 5: Calculate the aggregated preference, $\pi(\mathrm{Ma}$, $\mathrm{Mb}$ ) using Equation 9 as shown below.

$$
\pi(\mathrm{Ma}, \mathrm{Mb})=\frac{\left[\sum_{\mathrm{j}=1}^{\mathrm{n}} \mathrm{w}_{\mathrm{j}} \mathrm{P}_{\mathrm{j}}(\mathrm{Ma}, \mathrm{Mb})\right]}{\sum_{\mathrm{j}=1}^{\mathrm{n}} \mathrm{w}_{\mathrm{j}}} .
$$

The summation of the criteria weightages is always equal to 1; hence, the denominator of Equation 9 becomes one. Now, multiplying all the criteria weightages with their respective column elements of Table 11 and using Equation 9, find all aggregated preference values $\pi$ (Ma, $\mathrm{Mb}$ ).

Table 12 presents the calculated aggregated preference values.

Table 12. Calculating the aggregated preference, $\pi(\mathrm{Ma}, \mathrm{Mb})$

(Source: Own elaboration)

\begin{tabular}{|c|c|c|c|c|c|c|c|c|}
\hline Weights & 0.37657 & 0.09395 & 0.04529 & 0.21594 & 0.16932 & 0.07647 & 0.02247 & 1 \\
\hline & & & & & & & & $\begin{array}{l}\text { Aggregated } \\
\text { preference, } \\
\pi(\mathrm{Ma}, \mathrm{Mb})\end{array}$ \\
\hline \multicolumn{9}{|c|}{ Model 1} \\
\hline$\pi(\mathrm{M} 1, \mathrm{M} 2)$ & 0 & 0 & 0 & 0 & 0 & 0.06554 & 0 & 0.06554 \\
\hline$\pi(\mathrm{M} 1, \mathrm{M} 3)$ & 0 & 0 & 0 & 0 & 0 & 0.02185 & 0 & 0.02185 \\
\hline$\pi(\mathrm{M} 1, \mathrm{M} 4)$ & 0 & 0 & 0 & 0 & 0 & 0.07647 & 0 & 0.07647 \\
\hline$\pi(\mathrm{M} 1, \mathrm{M} 5)$ & 0 & 0 & 0 & 0 & 0 & 0 & 0 & 0 \\
\hline$\pi(\mathrm{M} 1, \mathrm{M} 6)$ & 0 & 0 & 0 & 0 & 0 & 0.04370 & 0 & 0.04370 \\
\hline \multicolumn{9}{|c|}{ Model 2} \\
\hline$\pi(\mathrm{M} 2, \mathrm{M} 1)$ & 0.18828 & 0.04697 & 0.01510 & 0 & 0.11288 & 0 & 0 & 0.36323 \\
\hline$\pi(\mathrm{M} 2, \mathrm{M} 3)$ & 0 & 0 & 0 & 0 & 0 & 0 & 0 & 0 \\
\hline$\pi(\mathrm{M} 2, \mathrm{M} 4)$ & 0 & 0 & 0 & 0 & 0 & 0.01092 & 0 & 0.01092 \\
\hline$\pi(\mathrm{M} 2, \mathrm{M} 5)$ & 0 & 0 & 0 & 0 & 0 & 0 & 0 & 0 \\
\hline$\pi(\mathrm{M} 2, \mathrm{M} 6)$ & 0.18828 & 0.04697 & 0 & 0 & 0 & 0 & 0 & 0.23526 \\
\hline \multicolumn{9}{|c|}{ Model 3} \\
\hline$\pi(\mathrm{M} 3, \mathrm{M} 1)$ & 0.18828 & 0.09395 & 0.04529 & 0.10797 & 0.11288 & 0 & 0.00749 & 0.55586 \\
\hline$\pi(\mathrm{M} 3, \mathrm{M} 2)$ & 0 & 0.04697 & 0.03019 & 0.10797 & 0 & 0.04370 & 0.00749 & 0.23632 \\
\hline$\pi(\mathrm{M} 3, \mathrm{M} 4)$ & 0 & 0 & 0 & 0 & 0 & 0.05462 & 0 & 0.05462 \\
\hline$\pi(\mathrm{M} 3, \mathrm{M} 5)$ & 0 & 0.04697 & 0 & 0 & 0 & 0 & 0 & 0.04697 \\
\hline$\pi(\mathrm{M} 3, \mathrm{M} 6)$ & 0.18828 & 0.09395 & 0.03019 & 0.10797 & 0 & 0.02185 & 0.00749 & 0.44973 \\
\hline \multicolumn{9}{|c|}{ Model 4} \\
\hline$\pi(\mathrm{M} 4, \mathrm{M} 1)$ & 0.37657 & 0.09395 & 0.04529 & 0.21594 & 0.16932 & 0 & 0.02247 & 0.92353 \\
\hline$\pi(\mathrm{M} 4, \mathrm{M} 2)$ & 0.18828 & 0.04697 & 0.03019 & 0.21594 & 0.05644 & 0 & 0.02247 & 0.56030 \\
\hline$\pi(\mathrm{M} 4, \mathrm{M} 3)$ & 0.18828 & 0 & 0 & 0.10797 & 0.05644 & 0 & 0.01498 & 0.36767 \\
\hline$\pi(\mathrm{M} 4, \mathrm{M} 5)$ & 0.18828 & 0.04697 & 0 & 0.10797 & 0.05644 & 0 & 0 & 0.39967 \\
\hline$\pi(\mathrm{M} 4, \mathrm{M} 6)$ & 0.37657 & 0.09395 & 0.03019 & 0.21594 & 0.05644 & 0 & 0.02247 & 0.79555 \\
\hline
\end{tabular}


Table 12. Calculating the aggregated preference, $\pi$ (Ma, Mb), cont.

(Source: Own elaboration)

\begin{tabular}{|c|c|c|c|c|c|c|c|c|}
\hline Weights & 0.37657 & 0.09395 & 0.04529 & 0.21594 & 0.16932 & 0.07647 & 0.02247 & 1 \\
\hline \multicolumn{7}{|c|}{ Model 5 } \\
\hline \multicolumn{7}{|c|}{$\begin{array}{c}\text { Aggregated } \\
\text { preference, } \\
\text { (Ma, Mb) }\end{array}$} \\
\hline$\pi$ (M5, M1) & 0.18828 & 0.04697 & 0.04529 & 0.10797 & 0.11288 & 0 & 0.02247 & 0.52386 \\
\hline$\pi$ (M5, M2) & 0 & 0 & 0.03019 & 0.10797 & 0.00000 & 0.06554 & 0.02247 & 0.22617 \\
\hline$\pi$ (M5, M3) & 0 & 0 & 0 & 0 & 0 & 0.02185 & 0.01498 & 0.03683 \\
\hline$\pi$ (M5, M4) & 0 & 0 & 0 & 0 & 0 & 0.07647 & 0 & 0.07647 \\
\hline$\pi$ (M5, M6) & 0.18828 & 0.04697 & 0.03019 & 0.10797 & 0 & 0.04370 & 0.02247 & 0.43958 \\
\hline \multicolumn{7}{|c|}{ Model 6 } \\
\hline$\pi$ (M6, M1) & 0 & 0 & 0.01510 & 0 & 0.11288 & 0 & 0 & 0.12798 \\
\hline$\pi$ (M6, M2) & 0 & 0 & 0 & 0 & 0 & 0.02185 & 0 & 0.02185 \\
\hline$\pi$ (M6, M3) & 0 & 0 & 0 & 0 & 0 & 0 & 0 & 0 \\
\hline$\pi$ (M6, M4) & 0 & 0 & 0 & 0 & 0 & 0.03277 & 0 & 0.03277 \\
\hline$\pi$ (M6, M5) & 0 & 0 & 0 & 0 & 0 & 0 & 0 & 0 \\
\hline
\end{tabular}

Step 6: Create an aggregate preference function matrix. Depending on the number of alternatives $m$, $m \times m$ matrix is formed. In this present analysis,
6 alternatives are considered so $6 \times 6$ matrix is formed as shown in Table 13 .

Table 13. Aggregate preference function matrix (Source: Own elaboration)

\begin{tabular}{|c|c|c|c|c|c|c|}
\hline Models & Model 1 & Model 2 & Model 3 & Model 4 & Model 5 & Model 6 \\
\hline Model 1 & - & 0.06554 & 0.02185 & 0.07647 & 0 & 0.04370 \\
\hline Model 2 & 0.36323 & - & 0 & 0.01092 & 0 & 0.23526 \\
\hline Model 3 & 0.55586 & 0.23632 & - & 0.05462 & 0.04697 & 0.44973 \\
\hline Model 4 & 0.92353 & 0.56030 & 0.36767 & - & 0.39967 & 0.79555 \\
\hline Model 5 & 0.52386 & 0.22617 & 0.03683 & 0.07647 & - & 0.43958 \\
\hline Model 6 & 0.12798 & 0.02185 & 0 & 0.03277 & 0 & - \\
\hline
\end{tabular}

For $\pi(\mathrm{M} 1, \mathrm{M} 2)$, the aggregated preference value is 0.06554; this means the aggregated preference value of Model 1 with respect to Model 2 is 0.06554 (from Table 12). So this value (i.e., 0.06554) is allotted in the cell 1, 2 as shown in Table 13.

Similarly, for $\pi$ (M1, M3), the aggregated preference value of Model 1 with respect to Model 3 is 0.02185 (from Table 12), so this value (i.e., 0.02185) is allotted in the cell 1, 3 as shown in Table 13.

In this way, the aggregated preference function value from Table 12 is allotted in all the cells of the above matrix, thus forming Table 13. When an alternative is compared to itself, no values should be assigned.
Step 7: Determination of the leaving and entering outranking flow of the alternatives.

The leaving and entering outranking flow in case of PROMETHEE I is calculated and presented in Table 14 using Equations 10 and 11 given below.

Leaving (positive) flow for ath alternative, $\varphi^{+}$,

$$
\sum_{\mathrm{b}=1}^{\mathrm{m}} \pi(\mathrm{a}, \mathrm{b})
$$

Entering (negative) flow for $\mathrm{a}_{\text {th }}$ alternative, $\varphi^{-}$,

$$
\sum_{\mathrm{b}=1}^{\mathrm{m}} \pi(\mathrm{a}, \mathrm{b})
$$

for example, $a \neq b$. 
The leaving and entering outranking flow in case of PROMETHEE II is calculated and presented in Table 15 using the Equations 12 and 13 given below.

Leaving (positive) flow for $a_{\text {th }}$ alternative, $\varphi^{+}$,

$$
\frac{1}{n-1} \sum_{b=1}^{m} \pi(a, b)
$$

Entering (negative) flow for ath alternative, $\varphi^{-}$,

$$
\frac{1}{n-1} \sum_{b=1}^{m} \pi(a, b)
$$

for example, $\mathrm{a} \neq \mathrm{b}$ and $\mathrm{n}$ is the number of alternatives.

Table 14. Determination of leaving and entering outranking flow (PROMETHEE I)

(Source: Own elaboration)

\begin{tabular}{|c|c|c|c|c|c|c|c|}
\hline Models & Model 1 & Model 2 & Model 3 & Model 4 & Model 5 & Model 6 & $\begin{array}{c}\text { Leaving } \\
\text { flow } \varphi^{+}\end{array}$ \\
\hline Model 1 & - & 0.06554 & 0.02185 & 0.07647 & 0 & 0.04370 & 0.20756 \\
\hline Model 2 & 0.36323 & - & 0 & 0.01092 & 0 & 0.23526 & 0.60942 \\
\hline Model 3 & 0.55586 & 0.23632 & - & 0.05462 & 0.04697 & 0.44973 & 1.34350 \\
\hline Model 4 & 0.92353 & 0.56030 & 0.36767 & - & 0.39967 & 0.79555 & 3.04672 \\
\hline Model 5 & 0.52386 & 0.22617 & 0.03683 & 0.07647 & - & 0.43958 & 1.30291 \\
\hline Model 6 & 0.12798 & 0.02185 & 0 & 0.03277 & 0 & - & 0.18260 \\
\hline Entering flow $\varphi-$ & 2.49446 & 1.11018 & 0.42635 & 0.25125 & 0.44664 & 1.96382 & \\
\hline
\end{tabular}

Table 15. Determination of leaving and entering outranking flow (PROMETHEE II)

(Source: Own elaboration)

\begin{tabular}{|c|c|c|c|c|c|c|c|}
\hline Models & Model 1 & Model 2 & Model 3 & Model 4 & Model 5 & Model 6 & $\begin{array}{c}\text { Leaving } \\
\text { flow } \varphi^{+}\end{array}$ \\
\hline Model 1 & - & 0.06554 & 0.02185 & 0.07647 & 0.00000 & 0.04370 & 0.04151 \\
\hline Model 2 & 0.36323 & - & 0.00000 & 0.01092 & 0.00000 & 0.23526 & 0.12188 \\
\hline Model 3 & 0.55586 & 0.23632 & - & 0.05462 & 0.04697 & 0.44973 & 0.26870 \\
\hline Model 4 & 0.92353 & 0.56030 & 0.36767 & - & 0.39967 & 0.79555 & 0.60934 \\
\hline Model 5 & 0.52386 & 0.22617 & 0.03683 & 0.07647 & - & 0.43958 & 0.26058 \\
\hline Model 6 & 0.12798 & 0.02185 & 0.00000 & 0.03277 & 0.00000 & - & 0.03652 \\
\hline Entering flow $\varphi-$ & 0.49889 & 0.22204 & 0.08527 & 0.05025 & 0.08933 & 0.39276 & \\
\hline
\end{tabular}

Step 8: Calculate the net outranking flow of each alternative using Equation 14. The net outranking flow of the alternatives are determined only in case of PROMETHEE II for the complete ranking of the alternatives. The net outranking flow of the alternatives is presented Table 16 .

Net Flow $\{\varphi(a)\}=$ Leaving Flow $\left\{\varphi^{+}(a)\right\}$

- Entering Flow $\left\{\varphi^{-}(\mathrm{a})\right\}$

Table 16. Net outranking flow of the alternatives (Source: Own elaboration)

\begin{tabular}{|c|c|c|c|}
\hline Models & Leaving flow $\varphi^{+}$ & Entering flow $\varphi^{-}$ & Net flow $\varphi$ \\
\hline Model 1 & 0.04151 & 0.49889 & -0.45738 \\
\hline Model 2 & 0.12188 & 0.22204 & -0.10015 \\
\hline Model 3 & 0.26870 & 0.08527 & 0.18343 \\
\hline Model 4 & 0.60934 & 0.05025 & 0.55909 \\
\hline Model 5 & 0.26058 & 0.08933 & 0.17125 \\
\hline Model 6 & 0.03652 & 0.39276 & -0.35624 \\
\hline
\end{tabular}




\section{$4 \quad$ Results and Discussions}

The best alternatives chosen by PROMETHEE method depends on the leaving and entering outranking flows of each alternatives, which are calculated in Section 3 and presented in Table 14 for PROMETHEE I and in Table 15 for PROMETHEE II.

In case of PROMETHEE II, the net outranking flow is also calculated as shown in Table 16.

As mentioned earlier, PROMETHEE 1 provides the partial ranking and PROMETHEE II provides the complete ranking of the alternatives. The outcome results from both the methods are explained in Sections 4.1 and 4.2, respectively.

\subsection{Outcome Results from PROMETHEE I}

In case of PROMETHEE I, partial ranking is performed and the decision made by the decision maker is based on the three conditions given below.

In PROMETHEE I, instead of defining the rank, preferences are calculated.
Condition 1: Alternative a is preferred to/outranks alternative $\mathrm{b}, \mathrm{aPb}$

$\mathrm{aPb}$ if:

$$
\begin{aligned}
& \varphi^{+}(a)>\varphi^{+}(b) \text { and } \varphi^{-}(a)<\varphi^{-}(b) \text {; or } \\
& \varphi^{+}(a)>\varphi^{+}(b) \text { and } \varphi^{-}(a)=\varphi^{-}(b) \text {; or } \\
& \varphi^{+}(a)=\varphi^{+}(b) \text { and } \varphi^{-}(a)<\varphi^{-}(b)
\end{aligned}
$$

Condition 2: Indifference situation, alb

aIb if:

$$
\varphi^{+}(\mathrm{a})=\varphi^{+}(\mathrm{b}) \text { and } \varphi^{-}(\mathrm{a})=\varphi^{-}(\mathrm{b})
$$

Condition 3: Incomparable situation, $\mathrm{aRb}$

$\mathrm{aRb}$ if:

$$
\begin{aligned}
& \varphi^{+}(\text {a })>\varphi^{+}\left(\text {b) and } \varphi^{-}(a)>\varphi^{-}\right. \text {(b); or } \\
& \varphi^{+}(\text {a })<\varphi^{+} \text {(b) and } \varphi^{-} \text {(a) }<\varphi^{-} \text {(b) }
\end{aligned}
$$

On the basis of the above three conditions, all the alternatives are compared among each other and the decisions are made comparing one laptop model over another, which is presented in Table 17.

As there are 6 alternatives selected for this research purposes, there will be 15 comparisons in total. Table 17 presents the choices and preferences of one model over another.

Table 17. Comparisons of the laptop models among each other (Source: Own elaboration)

\begin{tabular}{|c|c|c|c|}
\hline Sl. No. & Comparisons & Sl. No. & Comparisons \\
\hline 1 & Model 2 P Model 1 & 9 & Model 2 P Model 6 \\
\hline 2 & Model 3 P Model 1 & 10 & Model 4 P Model 3 \\
\hline 3 & Model 4 P Model 1 & 11 & Model 3 P Model 5 \\
\hline 4 & Model 5 P Model 1 & 12 & Model 3 P Model 6 \\
\hline 5 & Model 1 R Model 6 & 13 & Model 4 P Model 5 \\
\hline 6 & Model 3 P Model 2 & 14 & Model 4 P Model 6 \\
\hline 7 & Model 4 P Model 2 & 15 & Model 5 P Model 6 \\
\hline 8 & Model 5 P Model 2 & & \\
\hline
\end{tabular}

\subsection{Outcome Results from PROMETHEE II}

Ranking is performed according to the decreasing order of net flow values. Table 18 shows the com- plete ranking of the laptop models. The preference ranking order of the models are given as follows:

Model $4>$ Model $3>$ Model $5>$

Model $2>$ Model $6>$ Model 1 
Table 18. Ranking of the laptop models (Source: Own elaboration)

\begin{tabular}{|c|c|c|}
\hline Models & Net flow $\varphi$ & Ranking \\
\hline Model 1 & -0.45738 & Rank 6 \\
\hline Model 2 & -0.10015 & Rank 4 \\
\hline Model 3 & 0.18343 & Rank 2 \\
\hline Model 4 & 0.55909 & Rank 1 \\
\hline Model 5 & 0.17125 & Rank 3 \\
\hline Model 6 & -0.35624 & Rank 5 \\
\hline
\end{tabular}

\section{Conclusions}

At the end of this analysis, it can be concluded that Model 4 is the best laptop model among these six available models followed by Model 3 and Model 5 . Model 4 is coming out with the highest net flow value, that is, 0.55909 , which enables it to occupy the rank 1 position, and similarly, Model 1 is coming out with the lowest net flow value, that is, -0.45738 , so it occupies the last position, which denotes that it is the worst model among these group.

Although seven main important criteria are considered for this analysis, there are other specifications, both technical and nontechnical, that can also be included along with these, for example, display resolution, service center, weight, customer support, and graphics card for making these decision-making problem more precise and accurate. However, the same problem can also be carried out by applying other MCDM methods and the results can be compared with these results.

\section{$6 \quad$ References}

[1] Adalı, E. and Işık, A., 2016. Air conditioner selection problem with COPRAS and ARAS methods. Manas Journal of Social Studies, 5(2), pp.124-138. https://pdfs.semanticscholar.org/ b1f9/9b4c8ff39137f5f1f044df9572e5f62331b3.p df?_ga $=2.4748531 .1193764185 .1565108694-$ 1821258113.1558350277

[2] Adalı, E. and Iş̧1k, A., 2017. The multi-objective decision making methods based on MULTIMOORA and MOORA for the laptop selection problem. International Journal of Industrial Engineering, 13, pp.229-237. https://link.springer. com/content/pdf/10.1007\%2Fs40092-016-01755.pdf
[3] Albadvi, A., Chaharsooghi, S.K. and Esfahanipour, A., 2007. Decision making in stock trading: An application of PROMETHEE. European Journal of Operational Research, 177(2), pp.673-683. https://doi.org/10.1016/j.ejor.2005. 11.022

[4] Alomoush, M, I., 2010. Multicriteria selection of optimal location of TCSC in a competitive energy market, Journal of Electrical Engineering, 61(3), pp.129-140. https://content.sciendo.com /view/journals/jee/61/3/article-p129.xml?rskey $=6$ Byy3L\&result $=4 \&$ tab_body $=$ pdf

[5] Ayhan, M.B., 2013. A fuzzy AHP for supplier selection problem: A case study in a gear motor company. International Journal of Managing Value and Supply Chains (IJMVSC), 4(3), pp.1123. https://arxiv.org/ftp/arxiv/papers/1311/1311. 2886.pdf

[6] Behzadian, M., Kazemzadeh, R.B Albadvi, A. and Aghdasi, M., 2010. PROMETHEE: A comprehensive literature review on methodologies and applications. European Journal of Operational Research, 200(1), pp.198-215. https://doi. org/10.1016/j.ejor.2009.01.021

[7] Bentes, A.V., Carneiro, J., Da Silva, J.F. and Kimura, H., 2012. Multidimensional assessment of organizational performance: Integrating BSC and AHP. Journal of Business Research, 65(12), pp.1790-1799. https://doi.org/10.1016/j.jbusres. 2011.10.039

[8] Bertolini, M., Braglia, M. and Carmignani, G., 2006. Application of the AHP Methodology in Making a Proposal for a Public Work Contract. International Journal of Project Management, 24, pp.422-430. https://doi.org/10.1016/j.ijpro man.2006.01.005

[9] Bhattacharyya, A. and Chakraborty, S., 2014. A DEA-TOPSIS-based approach for performance evaluation of Indian technical institutes. Deci- 
sion Science Letters, 3(3), pp.397-410. http://www.growingscience.com/dsl/Vol3/dsl_2 014_5.pdf

[10] Brans, J.P., 1982. L'ingénièrie de la décision; Elaboration d'instruments d'aide à la décision. La méthode PROMETHEE. Organized by Nadeau, R. and Landry, M., L'aide à la décision: Nature, Instruments et Perspectives d'Avenir, Québec, Canada, Presses de l’Université Laval, pp.183-213.

[11] Brans, J.P. and Mareschal, B., 1992. Promethee$\mathrm{V}$ - MCDM problems with segmentation constraints. Information systems and operational research, 30(2), pp.85-96. https://doi.org/10.1080/ 03155986.1992 .11732186

[12] Brans, J.P. and Mareschal, B., 1995. The PROMETHEE VI procedure. How to differentiate hard from soft multicriteria problems. Journal of Decision Systems, 4(3), pp.213-223. https:// doi.org/10.1080/12460125.1995.10511652

[13] Brans, J.P. and Mareschal, P., 1994. The PROMETHEE-GAIA decision support system for multicriteria investigations. journal of Investigation Operative, 4(2), pp.107-117.

[14] Brans, J.P., Mareschal, B. and Vincke, P., 1984. PROMETHEE: A new family of outranking methods in multicriteria analysis. In J.P. Brans, editor, Operational Research'84, North-Holland, Amsterdam, pp.477-490.

[15] Brans, J.P., Mareschal, B. and Vincke, P., 1986. How to select and how to rank projects: The PROMETHEE method. European Journal of Operational Research, 24(2), pp.228-238. https://doi.org/10.1016/0377-2217(86)90044-5

[16] Brans, J.P. and Vincke, P., 1985. A preference ranking organisation method: The PROMETHEE method for MCDM. Management Science, 31(6), pp.647-656

[17] Brauers, W.K.M. and Zavadskas, E.K., 2006. The MOORA method and its applications to privatization in a transition economy. Control and cybernetics, 35(2), pp.445-469. https://pdfs. semanticscholar.org/3cc0/3a4ced96c113cb8ea4b def4fb8c85c6429c4.pdf?_ga=2.82634006.12805 31091.1565892816-1821258113.1558350277

[18] Brauers, W.K.M. and Zavadskas, E.K., 2009. Multi objectives optimization with discrete alternatives on the basis of ratio analysis. Intellectual economics, 2(6), pp.30-41. https://www3 .mruni.eu/ojs/intellectual-economics/article/view $/ 1193 / 1142$

[19] Buckley, J.J., 1985. Fuzzy hierarchical analysis. Fuzzy Sets and Systems, 17(3), pp.233-247. https://doi.org/10.1016/0165-0114(85)90090-9.

[20] Bulut, E., Duru, O., Keçeci, T. and Yoshida, S., 2012. Use of consistency index, expert prioritization and direct numerical inputs for generic fuzzy-AHP modeling: A process model for shipping asset management. Expert Systems and Applications, 39(2), pp.1911-1923. https://doi.org/ 10.1016/j.eswa.2011.08.056

[21] Butowski, L., 2018. An integrated AHP and PROMETHEE approach to the evaluation of the attractiveness of European maritime areas for sailing tourism. Moravian Geographical Reports, 26(2), pp.135-148. https://content.sciendo. $\mathrm{com} /$ view/journals/mgr/26/2/article-p135.xml? rskey $=6$ Byy $3 L \&$ result $=3 \&$ tab_body $=$ pdf

[22] Büyüközkan, G. and Çifçi, G., 2012. A combined fuzzy AHP and fuzzy TOPSIS based strategic analysis of electronic service quality in healthcare industry. Expert Systems with Applications, 39(3), pp.2341-2354. https://doi.org/10. 1016/j.eswa.2011.08.061

[23] Chandrakar, R. and Limje, S., 2018. A hybrid of QFD and AHP-TOPSIS for Durg dumping waste projects. Journal of Project Management, 3(3), pp.143-150. http://www.growingscience.com /jpm/Vol3/jpm_2018_8.pdf

[24] Chang, D.Y., 1996. Applications of the extent analysis method on fuzzy AHP. European Journal of Operational Research, 95(3), pp.649-655. https://doi.org/10.1016/0377-2217(95)00300-2

[25] Celik, M., Deha, Er. I. and Ozok, A.F., 2009. Application of fuzzy extended AHP methodology on shipping registry selection: The case of Turkish maritime industry. Expert Systems with Applications, 36, pp.190-198. http://citeseerx. ist.psu.edu/viewdoc/download?doi=10.1.1.469.3 $751 \&$ rep=rep $1 \&$ type $=$ pdf

[26] Durán, O. and Aguilo, J., 2008. Computer-aided machine-tool selection based on a fuzzy-AHP approach. Expert Systems and Applications, 34(3), pp.1787-1794. https://doi.org/10.1016/ j.eswa.2007.01.046

[27] Geldermann, J., Spengler, T. and Rentz, O., 2000. Fuzzy outranking for environmental assessment. Case study: Iron and steel making in- 
dustry. Fuzzy Sets and Systems, 115(1), pp.4565. https://doi.org/10.1016/S0165-0114(99)000 21-4

[28] Goswami, S. and Mitra, S., 2020. Selecting the best mobile model by applying AHP-COPRAS and AHP-ARAS decision making methodology. International Journal of Data and Network Science, 4(1), pp.27-42. http://www.growing science.com/ijds/Vol4/ijdns_2019_30.pdf

[29] Goumas, M. and Lygerou, V., 2000. An extension of the PROMETHEE method for decision making in fuzzy environment: Ranking of alternative energy exploitation projects. European Journal of Operational Research, 123(3), pp.606-613. https://doi.org/10.1016/S0377-2217 (99)00093-4

[30] Holst, A., 2019, August 30. Global pc unit shipments 2006-2019. Statista. Retrieved from https://www.statista.com/statistics/273495/globa 1-shipments-of-personal-computers-since-2006/

[31] Hwang, C.L., Lai, Y.J. and Liu, T.Y., 1993. A new approach for multiple objective decision making. Computers and Operational Research, 20(8), pp.889-899. https://doi.org/10.1016/03050548(93)90109-V

[32] Hwang, C.L. and Yoon, K., 1981. Multiple Attributes Decision Making Methods and Applications. New York: Springer-Verlag.

[33] Jayant, A. and Sharma, J., 2018. A comprehensive literature review of MCDM techniques ELECTRE, PROMETHEE, VIKOR and TOPSIS applications in business competitive environment. International Journal of Current Research, 10(2), pp.65461-65477. https://pdfs. semanticscholar.org/b370/ba1d421bcc7738c4b6 9d9a31cb61186afbf3.pdf

[34] Kabir, G. and Sumi, R.S., 2014. Power substation location selection using fuzzy analytic hierarchy process and PROMETHEE: A case study from Bangladesh. Energy, 72, pp.717-730. https://doi.org/10.1016/j.energy.2014.05.098

[35] Kilic, H.S., Zaim, S. and Delen, D., 2015. Selecting the best ERP system for SMEs using a combination of ANP and PROMETHEE methods. Expert Systems with Applications, 42(5), pp.2343-2352. http://isiarticles.com/bundles/ Article/pre/pdf/46451.pdf

[36] Kolios, A., Mytilinou, V., Lozano-Minguez, E. and Salonitis, K., 2016. A comparative study of multiple criteria decision making methods under stochastic inputs. MDPI Journal of Energies, 9(566). https://www.mdpi.com/1996-1073/9/7 1566/htm\#B34-energies-09-00566

[37] Korhonen, P., Koskinen, L. and Voutilainen, R., 2006. A financial alliance compromise between executives and supervisory authorities. European Journal of Operational Research, 175(2), pp.1300-1310. https://doi.org/10.1016/j.ejor.20 05.06.033

[38] Kowalski, K., Stagl, S., Madlener, R. and Omann, I., 2009. Sustainable energy futures: Methodological challenges in combining scenarios and participatory multi-criteria analysis. $E u$ ropean Journal of Operational Research, 197(3), pp.1063-1074. https://doi.org/10.1016/j. ejor.2007.12.049

[39] Kucharski, A., 2014. Analysis of selected investment fund type ranking position - PROMETHEE method application. Przedsiębiorczość $i$ Zarzadanie, 15(1), pp.41-53. https://content.scie ndo.com/view/journals/eam/15/1/article-p41.xml ?rskey $=6$ Byy 3 L\&result $=1 \&$ tab_body $=$ pdf

[40] Lee, A.H.I., Chen, W.C. and Chang, C. J., 2008. A fuzzy AHP and BSC approach for evaluating performance of IT department in the manufacturing industry in Taiwan. Expert Systems with Applications, 34(1), pp.96-107. https://doi.org /10.1016/j.eswa.2006.08.022

[41] Maletic, D., Maletic, M., Lovrencic, V., AlNajjar, B. and Gomiscek, B., 2014. An application of analytic hierarchy process (AHP) and sensitivity analysis for maintenance policy selection. Organizacija. 47(3), pp.177-188. https://content.sciendo.com/view/journals/orga/4 7/3/article-p177.xml?rskey=kfwM8g\&result= 3\&tab_body=pdf

[42] Marzouk, M. and Abdelakder, E., 2019. On the use of multi-criteria decision making methods for minimizing environmental emissions in construction projects. Decision Science Letters, 8(4), pp.373-392. http://www.growingscience. com/dsl/Vol8/dsl_2019_16.pdf

[43] Melón, M.G., Aragonés, B.P. and Carmen, G.C.M., 2008. An AHP-based evaluation procedure for innovative educational projects: A faceto-face vs. computer-mediated case study. Ome$g a, 36(5)$, pp.754-765. https://doi.org/10.1016/j. omega.2006.01.005 
[44] Mitra, S. and Goswami, S.S., 2019a. Selection of the desktop computer model by AHP-TOPSIS hybrid MCDM methodology. International Journal of Research and Analytical Reviews, 6(1), pp.784-790. http://ijrar.com/upload_ issue/ijrar_issue_20542876.pdf

[45] Mitra, S. and Goswami, S.S., 2019b. Application of simple average weighting optimization method in the selection of best desktop computer model. Advanced Journal of Graduate Research, 6(1), pp.60-68. https://pdfs.semanticscholar. org/bc72/cb3b02e433f9a4f2ac73a8081c23f70f0 $5 \mathrm{e} 0 . \mathrm{pdf}$

[46] Mitra, S. and Kundu, S., 2017. Application of analytic hierarchy process for domestic refrigerator selection. International Journal of Emerging Technologies in Engineering Research, 5(12), pp.126-132. http://www.ijeter.ever science.org/Manuscripts/Volume-5/Issue-12/Vol -5-issue-12-M-21.pdf

[47] Mitra, S. and Kundu, S., 2018. Application of TOPSIS for best domestic refrigerator selection. International Journal of Research and Analytical Reviews, 5(3), pp.226-231. https://ijrar. com/upload_issue/ijrar_issue_1212.pdf

[48] Naserizade, S.S., Nikoo, M.R. and Montaseri, H., 2018. A risk-based multi-objective model for optimal placement of sensors in water distribution system. Journal of Hydrology, 557, pp.147159. https://doi.org/10.1016/j.jhydrol.2017. 12.028

[49] Ngai, E.W.T. and Chan, E.W.C., 2005. Evaluation of Knowledge Management Tools Using AHP. Expert System Applications, 29(4), pp.889-899. https://doi.org/10.1016/j.eswa.2005. 06.025

[50] Nikouei, M.A., Oroujzadeh, M. and MehdipourAtaei, S., 2017. The PROMETHEE multiple criteria decision making analysis for selecting the best membrane prepared from sulfonated poly(ether ketone)s and poly(ether sulfone)s for proton exchange membrane fuel cell. Energy, 119, pp.77-85. https://doi.org/10.1016/j.energy. 2016.12.052

[51] Nourbakhsh, Z. and Yousefi, H., 2017. Presenting a conceptual model of data collection to manage the groundwater quality. Journal of $\mathrm{Wa}$ ter and Land Development, 35(1), pp.149-160. https://content.sciendo.com/view/journals/jwld/3 5/1/article-p149.xml?rskey $=\mathrm{kfwM} 8 \mathrm{~g} \&$ result $=5 \&$ tab_body=pdf

[52] Peng, A.H. and Xiao, X.M., 2013. Material selection using PROMETHEE combined with analytic network process under hybrid environment. Materials and Design, 47, pp.643-652. https://doi.org/10.1016/j.matdes.2012.12.058

[53] Polat, G., 2016. Subcontractor selection using the integration of the AHP and PROMETHEE methods. Journal of Civil Engineering and Management, 22(8), pp.1042-1054. https:// journals.vgtu.lt/index.php/JCEM/article/view /1960/1576

[54] Polat, G., Damci, A. and Dermili, L., 2016. Urban renewal project selection using the integration of AHP and PROMETHEE approaches. Procedia Engineering, 164, pp.339-346. https://reader.elsevier.com/reader/sd/pii/S18777 05816339698?token=B741435AA44C25D079F 221DBE3E0C2193EFDF30753523526E1ABC1 3625755EFC5FC37F0F17B73ED3142ACD4D5 1C4BBDC.

[55] Qin, X.S., Huang, G.H., Chakma, A. and Nie, X.H., 2008. A MCDM-based expert system for climate-change impact assessment and adaptation planning. A case study for the Georgia basin, Canada. Expert Systems with Applications, 34(3), pp.2164-2179. https://doi.org/10.1016/ j.eswa.2007.02.024.

[56] Rostamzadeh, R. and Sofian, S., 2011. Prioritizing effective 7Ms to improve production systems performance using fuzzy AHP and fuzzy TOPSIS (case study). Expert Systems with Applications, 38(5), pp.5166-5177. https://doi.org/ 10.1016/j.eswa.2010.10.045

[57] Roy, B., 1968. Classement et choix en présence de points de vue multiples (la méthode ELECTRE). Revue Francaise d'Informatique et de Recherche Opérationelle (RIRO), 2(8), pp.57-75. http://www.numdam.org/article/RO_1968__2_1 _57_0.pdf

[58] Saaty, T.L., 1980. The Analytic Hierarchy Process. New York: McGraw-Hill.

[59] Saaty, T.L., 2001. Fundamentals of Decision Making and Priority Theory. Pittsburgh, Pennsylvania: RWS Publications.

[60] Saaty, T.L., 2008. Decision Making for Leaders: The Analytic Hierarchy Process for Decisions in 
a Complex World. Pittsburgh, Pennsylvania: RWS Publications.

[61] Saaty, T.L., 2010. Principia Mathematica Decernendi: Mathematical Principles of Decision Making. Pittsburgh, Pennsylvania: RWS Publications.

[62] Sri Krishna, S., Sri Nivasulu Readdy, A. and Vani, S., 2014. A new car selection in the market using TOPSIS. International Journal of Engineering Research and General Science, 2(4), pp.177-181. http://ijergs.org/files/documents/ANEW20.pdf

[63] Tsoutsos, T., Drandaki, M., Frantzeskaki, N., Iosifidis, E. and Kiosses, I., 2009. Sustainable energy planning by using multi-criteria analysis application in the island of crete. Energy Policy, 37(5), pp.1587-1600. https://www.researchgate. net/profile/Niki_Frantzeskaki/publication/46496 901_Sustainable_energy_planning_by_using_m ulti-criteria_analysis_application_in_the_island _of_Crete/links/56c5d53408ae736e7049255c/Su stainable-energy-planning-by-using-multi-crite ria-analysis-application-in-the-island-ofCrete.pdf

[64] Turcksin, L., Bernardini, A. and Macharis, C., 2011. A combined AHP-PROMETHEE approach for selecting the most appropriate policy scenario to stimulate a clean vehicle fleet. Procedia Social and Behavioral Sciences, 20, pp.954-965. https://doi.org/10.1016/j.sbspro. 2011.08.104

[65] Vaillancourt, K. and Waaub, J.P., 2004. Equity in international greenhouse gases abatement scenarios: A multi criteria approach. European Journal of Operational Research, 153(2), pp.489-505. https://doi.org/10.1016/S0377-2217 (03)00170-X

[66] Wang, J.J. and Yang, D.L., 2007. Using a hybrid multi-criteria decision aid method for information systems outsourcing. Computers \& Operations Research, 34, pp.3691-3700. https://doi.org/10.1016/j.cor.2006.01.017
[67] Yilmaz, B. and Dagdeviren, M., 2011. A combined approach for equipment selection: FPROMETHEE method and zero-one goal programming. Expert Systems with Applications, 38(9), pp.11641-11650. http://www.irantahgig.ir /wp-content/uploads/40014.pdf

[68] Yoon, K., 1987. A reconciliation among discrete compromise situations. Journal of Operational Research Society, 38 (3), pp.277-286. https://doi.org/10.1057/jors.1987.44

[69] Zadeh, L.A., 1965. Fuzzy sets. Information and Control, 8(3), pp.338-353. https://reader.elsevier .com/reader/sd/pii/S001999586590241X?token= 7E8F7BB90871609078C2FA8FB927AD9370F8 43E3F43209FCC3CD2B59D0E7D1AF1CC306 EBEE6BEB1B5A4AE6A475CFDFB4

[70] Zavadskas, E.K., Kaklauskas, A., Turskis, Z. and Tamošaitien, J., 2008. Selection of the effective dwelling house walls by applying attributes values determined at intervals. Journal of Civil Engineering and Management, 14(2), pp.85-93. https://pdfs.semanticscholar.org/5268/356a08cb 6df2830747c841a13a3e708f1421.pdf

[71] Zavadskas, E.K. and Turskis, Z., 2010. A new additive ratio assessment (ARAS) method in multicriteria decision-making. Technological and Economic Development of Economy, 16(2), pp.159-172. https://www.tandfonline.com/doi/ pdf/10.3846/tede.2010.10

[72] Zavadskas, E.K., Turskis, Z. and Vilutiene, T., 2010. Multiple criteria analysis of foundation instalment alternatives by applying Additive Ratio Assessment (ARAS) method. Archives of Civil and Mechanical Engineering, 10(3), pp.123-141. https://doi.org/10.1016/S1644-9665(12)60141-1

[73] Zhao, H., Peng, Y. and Li, W., 2013. Revised PROMETHEE II for improving efficiency in emergency response. Procedia Computer Science, 17, pp.181-188. https://doi.org/10.1016/ j.procs.2013.05.025. 